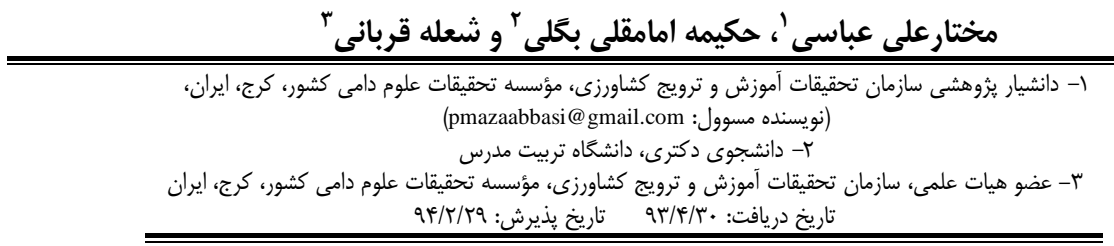

"ِكيده

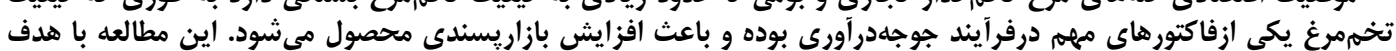

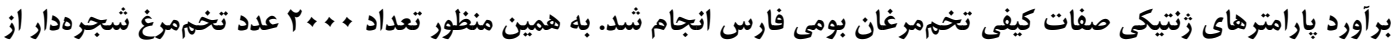

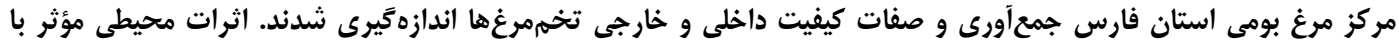

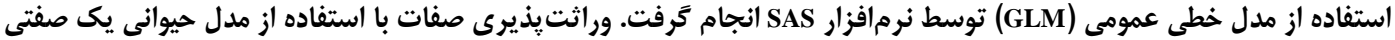

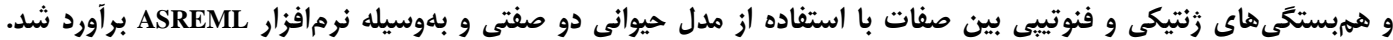

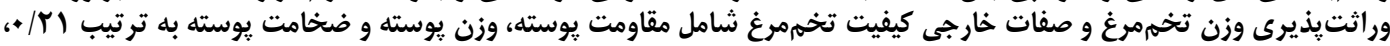

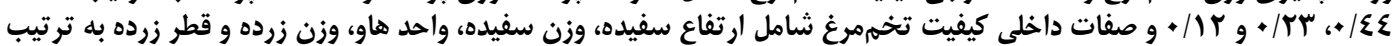

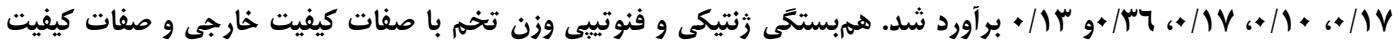

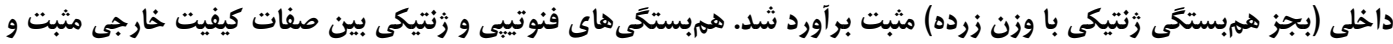

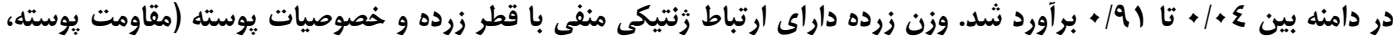

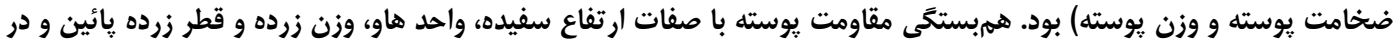

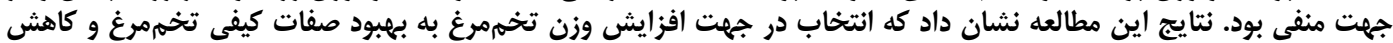

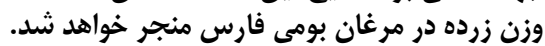

وازههاى كليدى: تخممرغ، كيفيت، مرغان بومى، مؤلفههاى (كو)واريانس

اين محصول را نيز افزايش مىدهد. صفات كيفى تخممرغ

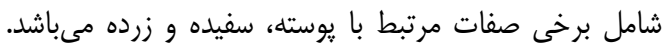

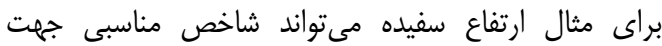

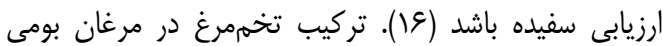

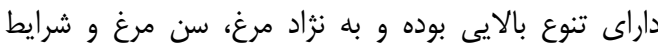

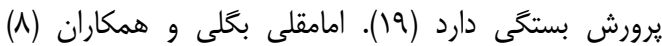

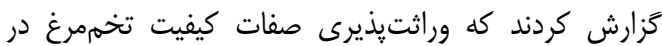

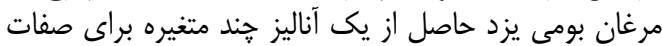

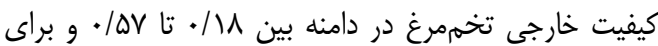

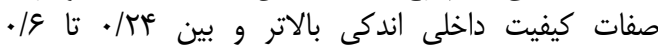

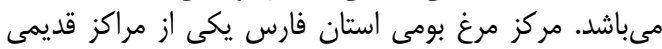

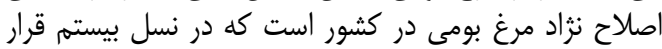

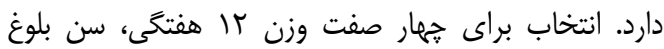

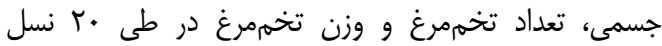

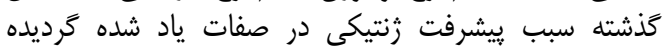

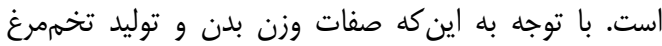

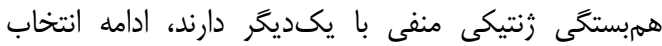

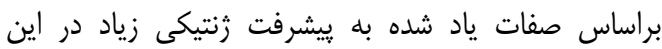

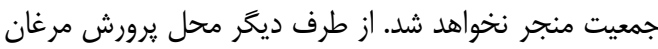

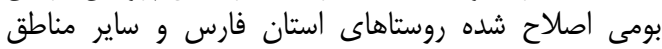

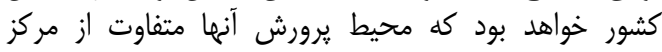

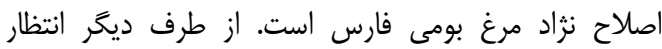

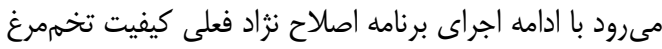
كاهش يابد و منظور كردن اين صفات در شاخص إنص انتخاب و
نزادهاى بومى كه حاصل هزاران سال انتخاب طبيعى

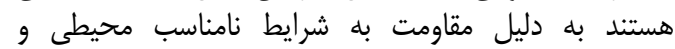

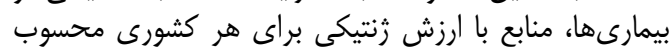

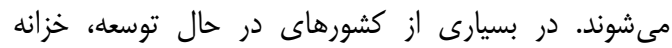

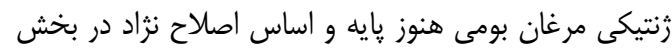

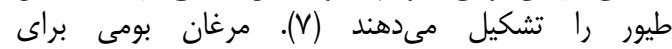
يرورشدهندكان سنتى در شهرهان

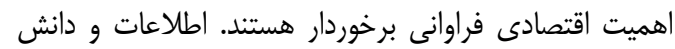

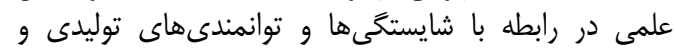

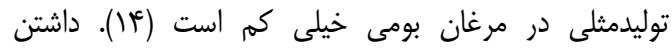

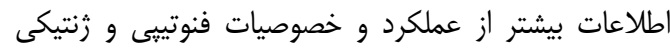

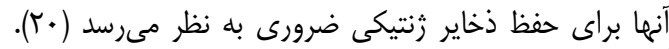

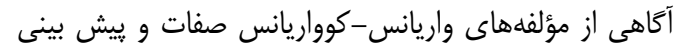

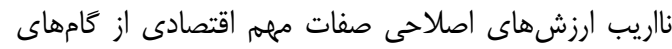

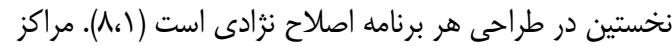

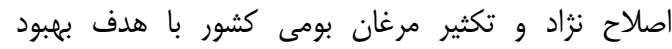

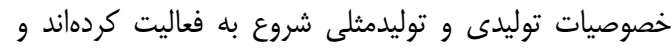

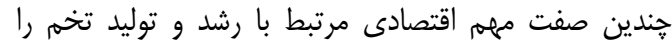
ركورديرى مى كنند (IV.V)

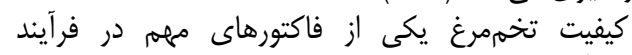

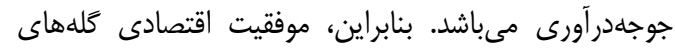

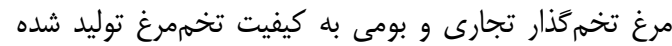

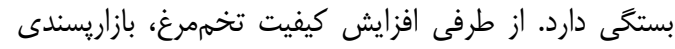


گرفتند. وراثتيذيرى صفات با استفاده از مدل حيوانى تك في

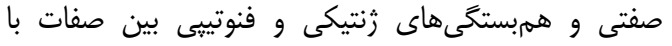

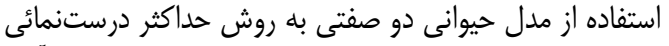

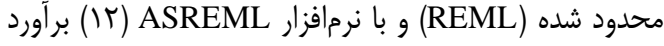

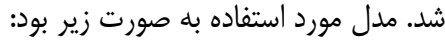
$\mathrm{y}_{\mathrm{i}}=\mathrm{X}_{\mathrm{i}} \mathrm{b}_{\mathrm{i}}+\mathrm{Z}_{\mathrm{i}} \mathrm{a}_{\mathrm{i}}+\mathrm{e}_{\mathrm{i}}$

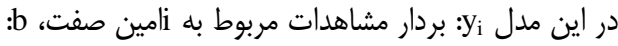

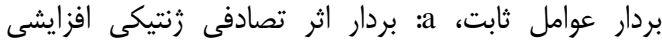

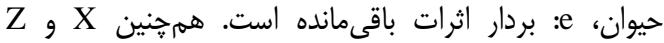

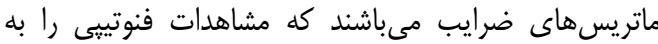
ترتيب به عوامل ثابت و تصادفى ربط مي ميدئ مهند.

نتايج و بحث ميان ميانكين صفات وحن

ميانخين و ضريب تغييرات صفات كيفيت خارجى و داخلى

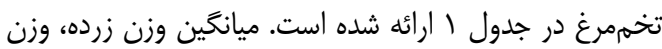

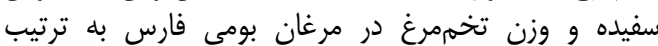

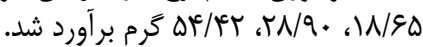

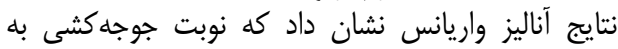

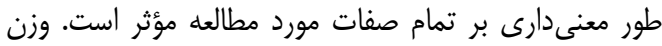

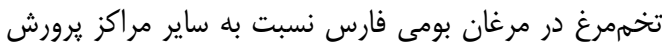

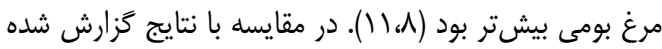

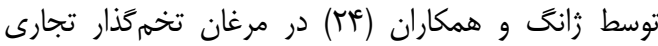

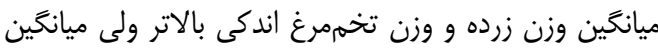

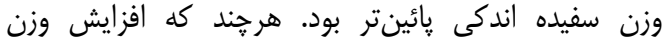

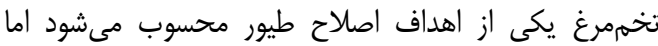

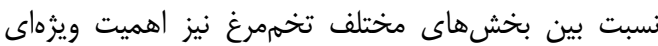

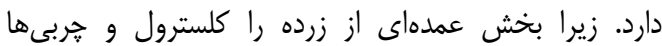

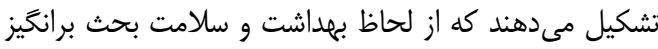

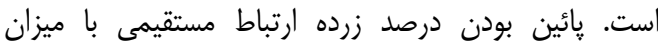

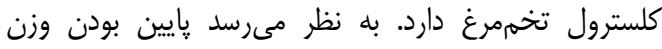

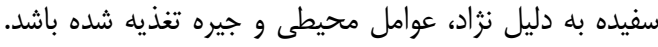

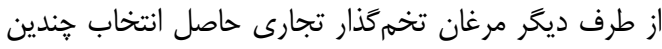

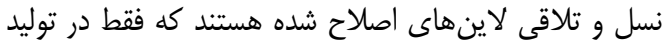

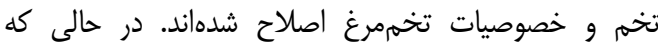

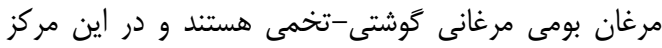

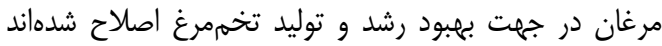

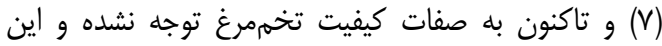

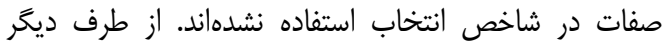

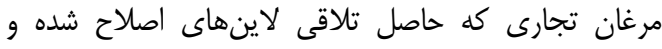

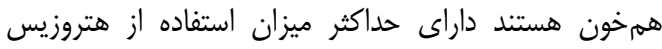

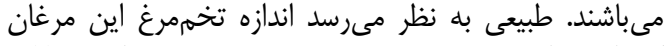

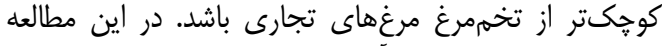

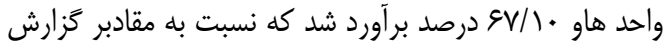

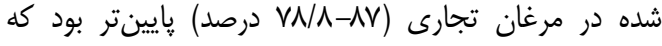

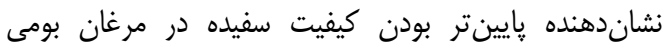

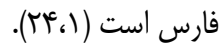

هدف اصلاح نزاد اين مركز حائز اهميت زيادى است. براى

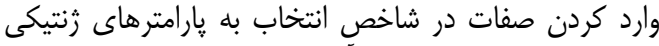

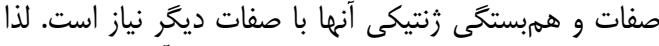

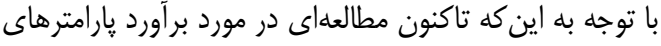

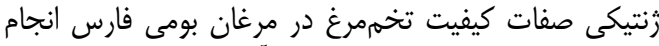

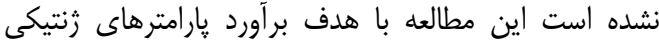

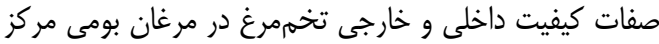

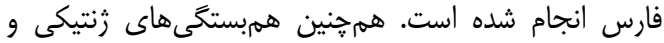

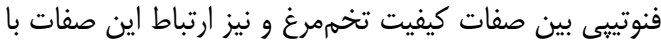
وزن تخممرغ برآورد شده است.

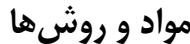

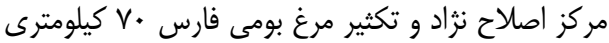

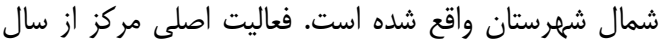

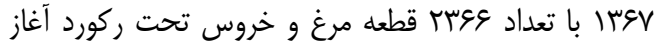

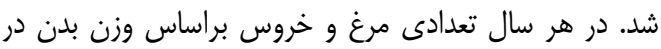

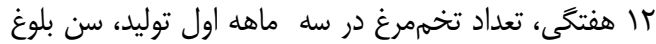

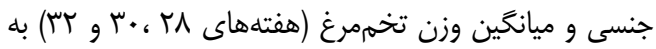

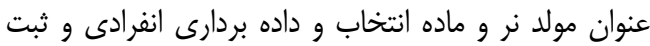
مشخصات شجرهاى انجام مى موداد

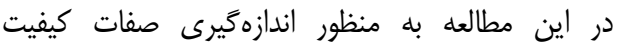

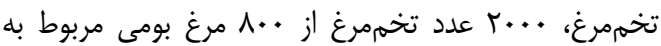

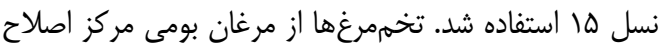

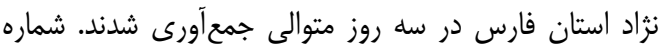

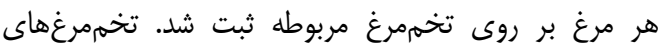

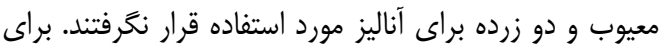

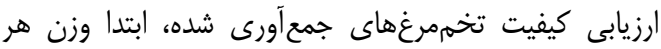

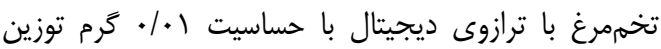

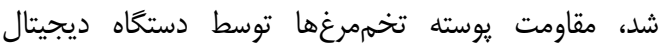

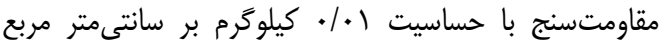

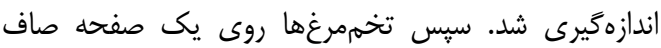

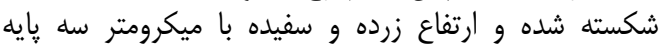

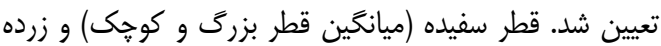

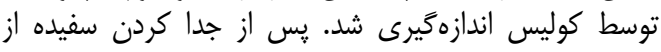

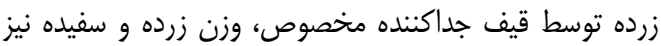

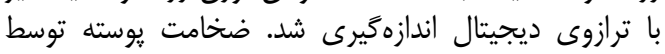

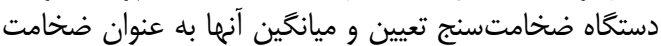

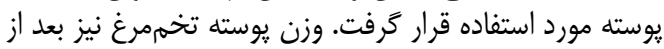

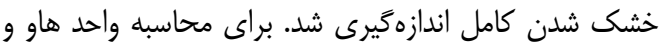

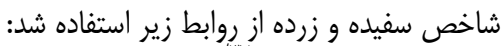

$\mathrm{HU}=1 \cdots \log (\mathrm{AH}-\mathrm{V} / \mathrm{VEW} / \mathrm{RV}+\mathrm{V} / \Delta \mathrm{V})$

$$
\text { شاخص سفيده }=\text { قرتفر سفيده سفيده }
$$

در رابطه فوق، HU: واحد هاو، EW: وزن تخممرغ و AH: ارتفاع سفيده مىباشد.

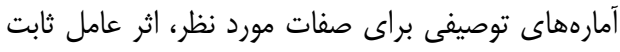

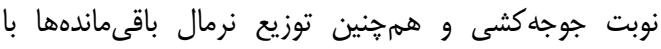
استفاده از نرمافزار SAS نسخه جنه 
Table 1. Means and coefficient of variations of studied traits

جدول (- ميانخين و ضريب تغييرات صفات مورد مطالعه

\begin{tabular}{|c|c|c|c|c|c|}
\hline \multicolumn{3}{|c|}{ كيفيت داخلى تخممرغ } & \multicolumn{3}{|c|}{ كيفيت خارجى تخممرغ } \\
\hline ضريب تغييرات (٪) & ميانكين & صفت & ضريب تغييرات & ميانكين & صفت \\
\hline$I V / N^{\mu}$ & $\Delta E / E T$ & وزن تخممرغ (كرم) & $4 / .1$ & $F / W V$ & ارتفاع سفيده (ميلىمتر) \\
\hline$\Gamma \Delta / \Delta V$ & ه/89 & طول تخممرغ (سانتىمتر) & $11 / 4$ & rN/q. & وزن سفيده (كرم) \\
\hline FW/ & e/r. & عرض تخممرغ (سانتىمتر) & $|Q / \Gamma|$ & W/9D & وزن زرده (كرم) \\
\hline$r / \mathrm{r}$ & $\cdot / V^{e}$ & شاخص تخممرغ & $9 / 4 \wedge$ & $9 \mathrm{~V} / 1$. & واحد هاو \\
\hline $\mid r / 9 T$ & $r / M$ & مقاومت يوسته ( (kg/cm) & $1 r / r q$ & 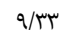 & قطر سفيده (سانتىمتر) \\
\hline $1 \% / 99$ & $\Delta / \Gamma r$ & وزن يوسته (كرم) & & q/r & قطر بزرى سفيده (سانتىمتر) \\
\hline $\mid f / \cdot F$ & ./\%q & ضخامت يوسته (ميلى متر) & r & $\Delta / \backslash \Lambda$ & شاخص سفيده (درصد) \\
\hline- & - & - & r & $F / l f$ & قطر زرده \\
\hline
\end{tabular}

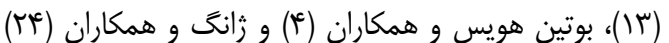

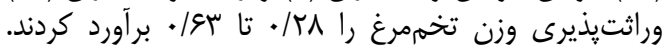

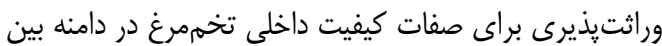

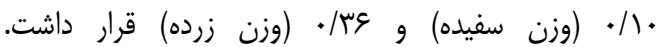
وراثتيذيرى صفات ضخامت يوسته، ارتفاع سفيده و و واحدان إناو

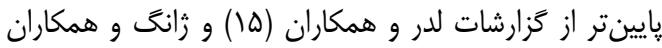

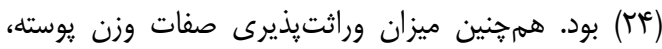

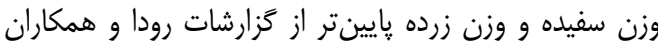

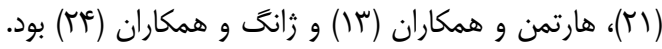

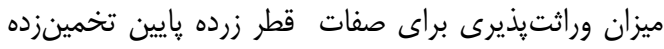

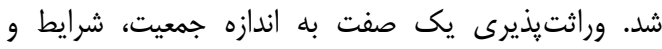

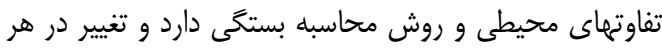

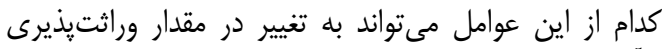

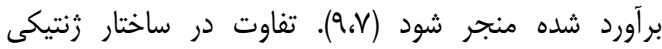

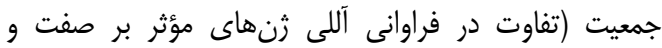

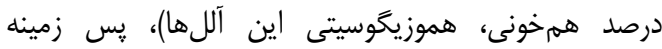

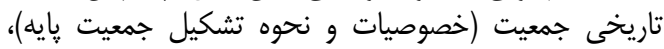

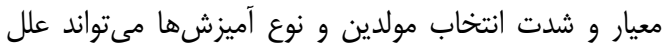

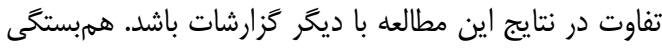

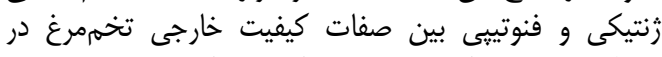

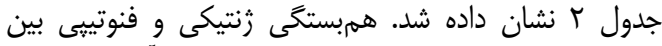

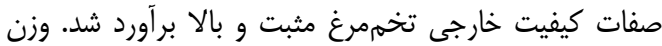

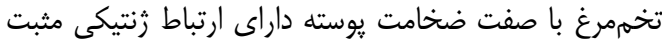

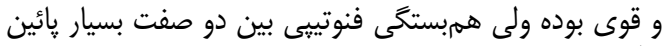

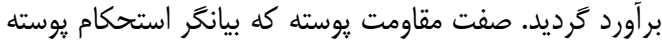

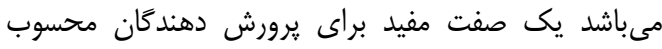

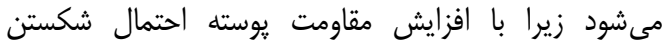

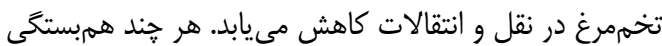

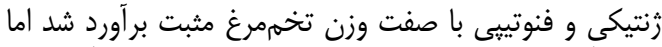

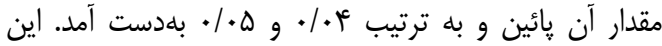

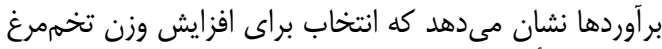

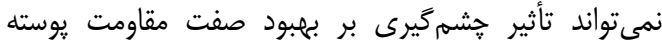

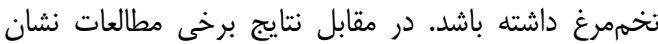

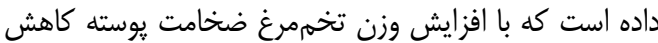

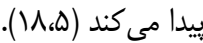

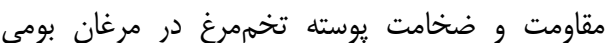

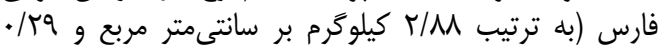

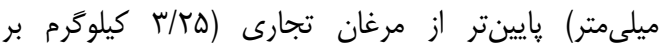

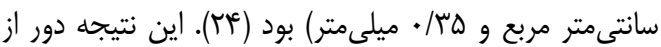

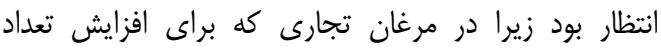

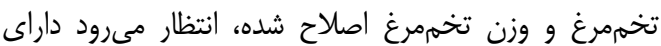

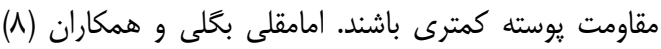

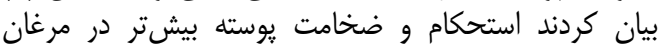
بومى بيان

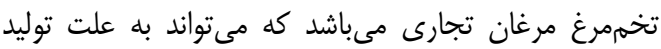

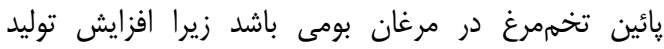

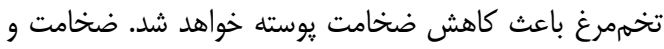

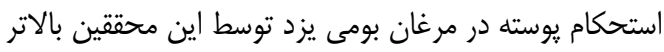

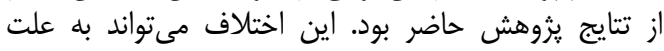

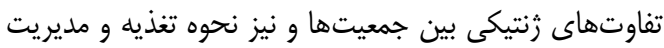

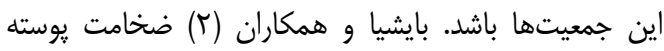

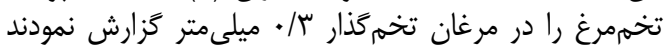

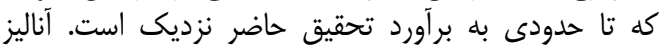

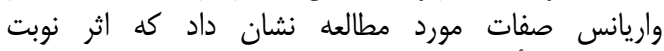

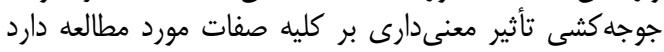

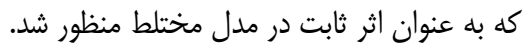

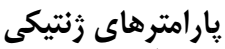

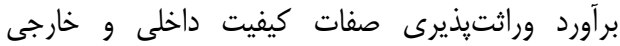

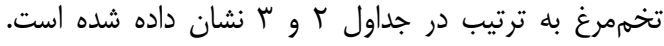

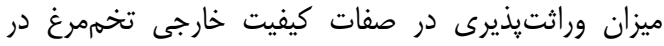

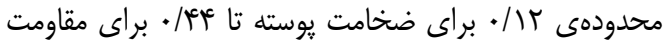

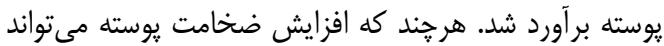

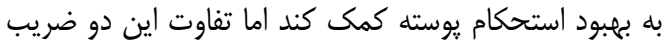

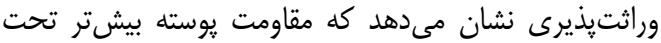

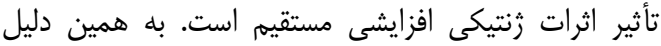

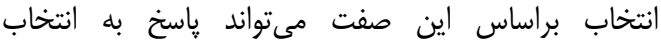

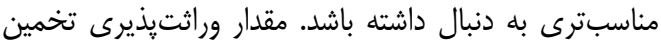

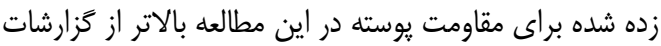

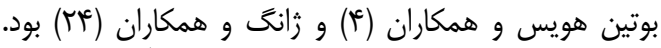

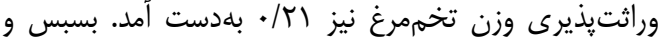
كيبسون (بّ)، فرانسيش و همكاران (•) (1)، هارتمن و همكاران 
جدول r- وراثتيذيرى (قطر جدول)، همبستخى زنتيكى (بالاى قطر) و همبستخى هاى فنوتيبى (زير قطر) به همراه خطاى استاندارد صفات

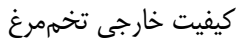

Table 2. Heritabilities (diagonal), genetic corelations (above diagonal) and phenotypic correlations (under diagonal) with their standard error of external egg quality traits

\begin{tabular}{|c|c|c|c|c|}
\hline ضخامت يوسته & وزن يوسته & مقاومت يوسته & وزن تخممرغ & صفات \\
\hline$\cdot|\Lambda T \pm . / \Gamma|$ & $\cdot|V| \pm \cdot|r|$ & $\cdot / \cdot 4 \pm \cdot / 1$. & $\cdot / r \mid \pm . / \cdot 9$ & وزن تخممرغ \\
\hline$. / 91 \pm \cdot / 1$. & $\cdot / \Lambda \mu \pm \cdot / \Lambda \Lambda$ & $\cdot / 4 x \pm \cdot / 1$ & $\cdot / \cdot \Delta \pm \cdot / \cdot r$ & مقاومت يوسته \\
\hline$\cdot / \Lambda \vee \pm \cdot / r$. & $. / r \mu \pm .1 \cdot 9$ & $\cdot / \kappa \Delta \pm \cdot / \cdot r$ & $\cdot / F^{V} V \pm \cdot / \cdot r$ & وزن يوسته \\
\hline$. / 1 r \pm . / .9$ & $\cdot / \& \Lambda \pm . / \cdot r$ & $\cdot / v 9 \pm . / \cdot 1$ & $. / .9 \pm . / . \%$ & ضخامت يوسته \\
\hline
\end{tabular}

جدول ب- وراثتيذيرى (قطر جدول)، همبستخى زنتيكى (بالاى قطر) و همبستخى هاى فنوتيبى (زير قطر) صفات كيفيت داخلى تخم مرغ Table 3. Heritabilities (diagonal), genetic corelations (above diagonal) and phenotypic correlations (under diagonal)

\begin{tabular}{|c|c|c|c|c|c|}
\hline قطر زرده & واحد هاو & وزن زرده & وزن سفيده & ارتفاع سفيده & صفات \\
\hline - & $\cdot / 99 \pm . / . r$ & $\cdot / \cdot \Delta \pm \cdot / I T$ & $\cdot|ধ 9 \pm \cdot / \pi|$ & $\cdot / / V \pm \cdot / \cdot V$ & ارتفاع سفيده \\
\hline$-\mathrm{ns}$ & $\cdot / V \pm \cdot / T V$ & سז/. & $.11 \cdot \pm \cdot 1 \cdot 9$ & r./IrE./. & وزن سفيده \\
\hline$-/ \Delta 9 \pm \cdot / \% q$ & $\cdot / N V \pm \cdot / r T$ & $\cdot / 4= \pm . / 1$. & r./ & . & وزن زرده \\
\hline$\cdot / N E \pm . / T r$ & $\cdot / \mid \gamma \pm \cdot / \cdot \Delta$ & $-\cdot / \cdot \Delta \pm \cdot / \cdot r$ & $\cdot / \cdot r \pm \cdot / \cdot r$ & $. / 94 \pm . / .$. & واحد هاو \\
\hline$\cdot / / r \pm \cdot / \cdot v$ & $\cdot / \cdot r \pm . / . r$ & $\cdot / 4 t \pm \cdot / \cdot r$ & -ns & $. / \cdot r \pm . / \cdot r$ & قطر زرده \\
\hline
\end{tabular}

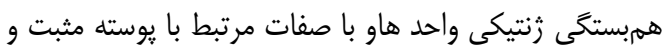

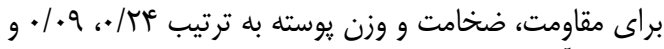

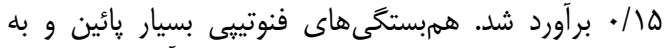

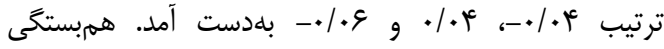
زنتيكى وزن تخممرغ بأ باء صفات كيفيت داخلى (بغير از وزن

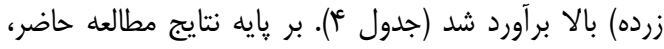

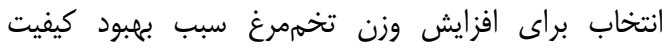

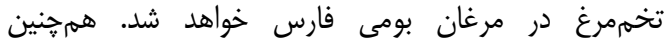

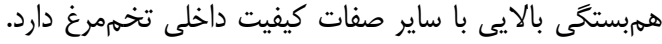

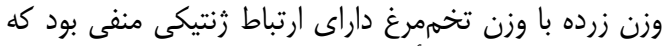

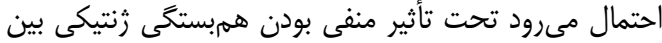

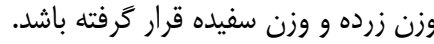

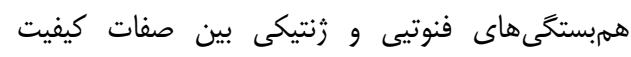

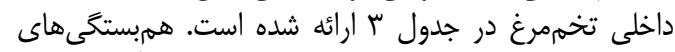

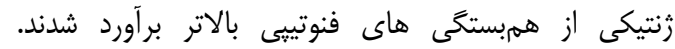

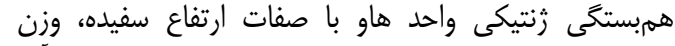

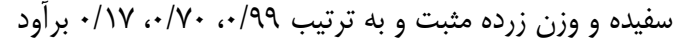

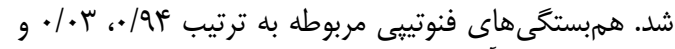

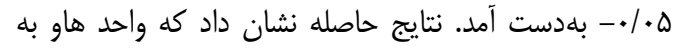

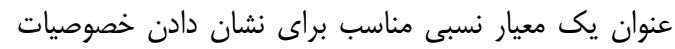

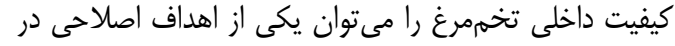

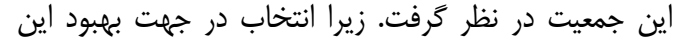

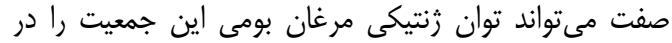

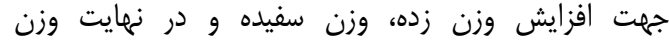
تخممرغ هاى توليدى ارتقاء بخشد.

جدول fا- همبستخى زنتيكى بين كيفيت داخلى و خارجى تخممرغ بههمراه خطاى استاندارد Table 4. Genetic correlations between internal and external egg quality traits with their standard errors

\begin{tabular}{|c|c|c|c|c|}
\hline وزن يوسته & ضخامت يوسته & مقاومت يوسته & وزن تخممرغ & صفات \\
\hline . $/ N \pm \cdot / r \mu$ & $\cdot / \backslash \Lambda \pm \cdot / R \mu$ & $. / K \varepsilon \pm \cdot / / Q$ & $. / 9 \mathrm{~V} \pm . / 19$ & ارتفاع سفيده \\
\hline$\cdot / \Delta V \pm \cdot / r T$ & $\cdot / \Delta \Psi \pm \cdot / \mu \cdot$ & $-. / \cdot 1 \pm . / \cdot r$ & $\cdot / \Lambda \vee \pm \cdot / / Q$ & وزن سفيده \\
\hline$\cdot / 1 \Delta \pm \cdot / r$. & $.1 .9 \pm .1 .1$ & $\cdot / r F \pm \cdot / T \Delta$ & $\cdot / \Lambda \Delta \pm \cdot / R \varphi$ & واحد هاو \\
\hline$-\cdot / \mu T \pm \cdot / r \mid$ & $-\cdot / r \Lambda \pm \cdot / 19$ & $-\cdot / \cdot 1 \pm \cdot / 19$ & $-\cdot / r \cdot \pm \cdot|r|$ & وزن زرده \\
\hline$\cdot / V \cdot \pm \cdot / r \cdot$ & -ns3 & $-\cdot / 9 \Delta \pm \cdot / T$. & -ns3 & قطر زرده \\
\hline
\end{tabular}

همبستخى هاى زنتيكى و فنوتيبى صفات نشان ميدهد كه در

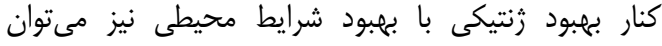
تخمرغ هايى با كيفيت بالا توليد كرد.

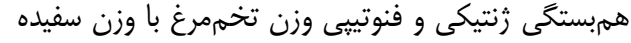

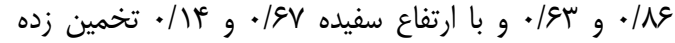

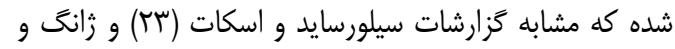

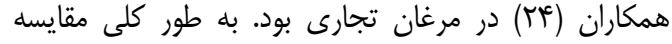

جدول ه- همبستخى فنوتيبى صفات خارجى و صفات داخلى تخممرغ به همر اه خطاى استاندارد Table 5. Phenotypic correlations between internal and external egg quality traits with their standard errors

\begin{tabular}{|c|c|c|c|c|}
\hline وزن يوسته & ضخامت يوسته & مقاومت يوسته & وزن تخممرغ & صفات \\
\hline$\cdot / \cdot V \pm \cdot / \cdot r$ & $\cdot / \cdot r \pm \cdot / \cdot r$ & $-\cdot / \cdot r \pm \cdot / \cdot \psi$ & $\cdot / N \pm \pm \cdot / \cdot r$ & ارتفاع سفيده \\
\hline$. / r \mu \pm .1 .9$ & $\cdot / \cdot \Lambda \pm \cdot / \cdot r$ & $. / \cdot \Delta \pm \cdot / \cdot F$ & $. / q \mu \pm . / \cdot r$ & وزن سفيده \\
\hline$-.1 .9 \pm .1 . r$ & $\cdot / \cdot r \pm \cdot / \cdot r$ & $-. / . r \pm . / . r$ & $\cdot / \cdot v \pm \cdot / \cdot r$ & واحد هاو \\
\hline$\cdot / \mid \varepsilon \pm . / \cdot \mu$ & $\cdot / 1 \cdot \pm \cdot / \cdot r$ & $-.1 .9 \pm .1 . \mu$ & $\cdot / r \Lambda \pm \cdot / \cdot r$ & وزن زرده \\
\hline$\cdot / \cdot V \pm \cdot / \cdot r$ & $-n s 3$ & $-. / 1 r \pm . / . r$ & -ns3 & قطر زرده \\
\hline
\end{tabular}

بنابراين لزوم توجه به بهبود كيفيت تخممرغ همزمان با ساير صفات ضرورى است.

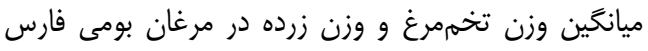

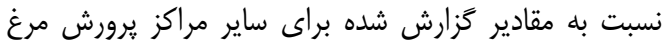

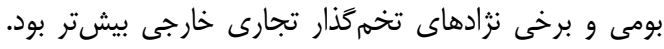


از اين صفات مىتواند ميانخين ساير صفات را در جمعيت بالا

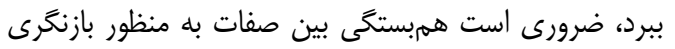

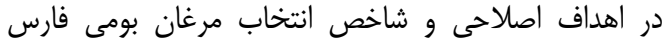

$$
\text { مورد استفاده قرار كيرد. }
$$

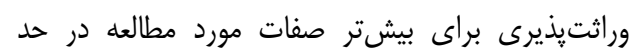

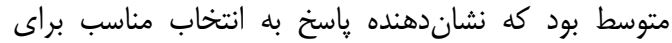

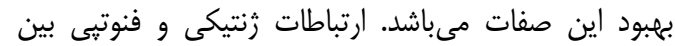

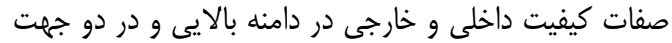

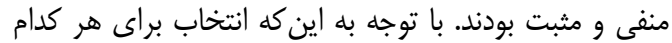

1. Abbasi, M.A. 2015. Effect of missing sire pedigree information on genetic trend and gain of quantitative trait using computer simulation. Research on Animal Production Vol. 6, No. 12, autumn and winter 2015, $152-159$.

2. Baishya, D., K.K. Dutta, J.D Mahanta and R.N. Borpujari. 2008. Studies on certain qualities of different sources of chicken eggs. Veterinary Animal Science, 4: 139-141.

3. Besbes, B. and J.P. Gibson. 1998. Genetic variability of egg production traits in purebred and crossbred laying hens. $6^{\text {th }}$ World Congress Genetic Applied Livestock Production, 25: 459-462.

4. Buitenhuis, A.J., T.B. Rodenburg, P.H. Wissink, J. Visscher, P. Koene, H. Bovenhuis, B.J. Ducro and J.J. Van Der Poel. 2004. Genetic and phenotypic correlations between feather pecking behavior stress response, immune response and egg quality traits in laying hEns. British Poultry, 83: 1077-1082.

5. De Ketelaere, B., T. Govaerts, P. Couke, E. Dewil, T. Visseher and L. Decuypere. 2002. Measuring the eggshell strength of 6 different strains of laying hens: Techniques and Comparison. British Poultry Science, 43: 238-244.

6. Emamgholi Begli, H., S. Zerehdaran, S. Hassani, M.A. Abbasi and A.K. Khan Ahmadi. 2010. Heritability, genetic and phenotypic correlations of egg quality traits in Iranian native fowl. British Poultry Science, 51: 740-744.

7. Emamgholi Begli, H., S. Zerehdaran, S. Hassani, A.K. Khan Ahmadi and M.A. Abbasi. 2010. Estimation of genetic and phenotypic correlations for performance and egg quality traits in native fowls of yazd province. Animal Science Researches, 24: 99-100.

8. Emamgholi Begli, H., S. Zerehdaran, S. Hassani and M.A. Abbasi. 2009. Estimation of genetic parameters of economically important traits in native fowl, Yazd Province. Iranian Journal of Animal Science, 40: 63-70.

9. Falconer, D.S. and T.F.C. Mackay. 1996. Introduction to quantitative genetics. $4^{\text {th }}$ edn. Longman. Group: Essex.

10. Francesch, A., J. Stony, L. Alfonso and M. Iglesias. 1997. Genetic parameters for egg number, egg weight, and egg shell color in three Catalan poultry breeds. Poultry Science, 76: 1627-1631.

11. Ghazikhan Shad, A., A. Nejati Javaremi and H. Mehrabani Yeganeh. 2007. Animal model estimation of genetic parameters for most important economic traits in Iranian native fowls. Biological Science, 10: 2787-2789.

12. Gilmour, A.R., B.R. Cullis, S.J. Welham and R. Thompson. 2000. ASREML. NSW Agriculture, Orange, Australia.

13. Hartmann, C., K. Johansson, E. Strandberg and L. Rydhmer. 2003. Genetic correlation between the maternal genetic effect on chick weight and direct genetic effects on egg composition traits in a White Leghorn line. Poultry Science, 82: 1-8.

14. Hoffman, I. 2005. Research and investment in poultry genetic resources-challenges and options for sustainable use. Journal of World's Poultry Science, 61: 57-70.

15. Ledur, M. C., L.E. Liljedahl, R.W. Fairfull, I. McMillan and L. Asselstine. 2002. Genetic effects of aging on egg quality traits in the first laying cycle of White Leghorn strains and strain crosses. Poultry Science, 81: 1439-1447.

16. Liljedahl, L.E., J.S. Gavora, R.W. Fairfull and R.S. Gowe. 1984. Age changes in genetic and environmental variation in laying hens. Theoretical and applied genetics, 67: 391-401.

17. Mohaghegh Dowlatabadai, M. R. 1999. Evaluation of economic traits of native fowls. M.Sc. Thesis, Tehran University, Karaj, Iran. 111 pp (In Persian).

18. Mohanty, S.C., H. Kanungo and M. Mishra. 1986. Effect of age at laying on the quality of egg of white leghorn hens. Indian Animal Production Management, 2: 184-186.

19. Narushin, V.A. and M.N. Romanov. 2002. Egg physical characteristics and hatchability. World's Poultry Science Journal, 58: 297-303.

20. Nikoubin Boroujeni, M., N. Pirany and F. Rafiei Boroujeni. 2016. Analysis of genetic diversity in fars native chicken based on partial mitochondrial dna d-loop region sequences. Research on Animal Production Vol. 7, No. 14, autumn and winter 2016, 180-185.

21. Rodda, D.D., G.W. Friars, J.S. Gavora and E.S. Meritt. 1977. Genetic parameter estimates and strain comparisons of egg compositional traits. British Poultry Science, 18: 459-473.

22. SAS Institute. 2001. SAS/STAT User's Guide: Statistics. Release 8.2. SAS Institute Inc., Cary, NC.

23. Silversides, F.G. and T.A. Scott. 2001. Effect of storage and layer age on quality of eggs from two lines of hens. Poultry Science, 80: 1240-1245.

24. Zhang, L.C., Z.H. Ning, G.Y. Xu, Z. Chou and N. Yang. 2005. Heritability and genetic and phenotypic correlations of egg quality traits in brown-egg dwarf layers. Poultry Science, 84: 1209-1213. 


\title{
Estimation of (Co)Variance Components of Egg Quality Traits for Fars Native Fowls
}

\author{
Mokhtar Ali Abbasi ${ }^{1}$, Hakimeh Emamgholi Begli ${ }^{2}$ and Sholeh Ghorbani ${ }^{3}$ \\ 1- Research Associate Research Organization of Agricultural Extension and Education, Animal Science \\ Research Institute, Karaj, Iran (Corresponding author: pmazaabbasi@gmail.com) \\ 2- PhD Student, University of Tarbiat Modarres \\ 3- Faculty Member, Research, Education and Extension Organizations, Animal Science Research Institute, \\ Karaj, Iran Animal Science Research Institute, Karaj, Iran \\ Received: July 21, 2014 \\ Accepted: May 19, 2015
}

\begin{abstract}
Economically success incomerical and Native laying hen farms in some eaten is related to egg quality. So, egg quality is a one of the most important factor affecting hatchability and lead to increasing the egg demand. The current study was carried out in aim to estimate the genetic parameters for egg quality traits in Fars native fowls. To do this, 2000 pedigree recorded eggs were collected at Fars Native Breeding Center and external and internal egg quality traits were measured. Significance of environmental effects determined using GLM procedure by SAS software and those were considered in the mixed model analysis. Heritability, genetic and phenotypic correlations were estimated using ASREML software. The estimated heritability for egg weight and external egg quality traits including shell strength, shell weight, shell thickness were $0.21,0.44,0.23,0.12$. this parameter for internal egg quality traits including albumin height, albumin weight, Hough unit, yolk weight, yolk diameter were also estimated 0.17, 0.10, $0.17,0.36,0.13$, respectively. Genetic and phenotypic correlations of egg weight with external and internal (except to yolk weight) egg quality traits were obtained positive. Both genetic and phenotypic correlations among external egg quality traits were found positive and ranged from 0.04 to 0.91 . Yolk weight were negatively genetically correlated with yolk diameter and shell features (shell strength, shell thickness and shell weight). The genetic correlation of shell strength and traits such as albumin height, Hough unit, yolk weight and yolk diameter were low and in negative direction. The results showed that the selection for increasing egg weight lead to improvement of egg quality traits and decreased yolk weight in Fars native fowls.
\end{abstract}

Keywords: (Co)Variance Components, Egg, Native Fowls, Quality 